\title{
COUPLING MECHANISM OF REGIONAL CARBON-WATER SYMBIOSIS SYSTEM AND WATER RESOURCES REGULATION AND CONTROL UNDER LOW CARBON PERSPECTIVE
}

\author{
XU, J. ${ }^{*}$ - CHEN, X.Y. - CHEN, X. \\ School of Management, Tianjin University of Technology, Tianjin 300384, China \\ *Corresponding author \\ e-mail:xujinbox@163.com \\ (Received $8^{\text {th }}$ Mar 2017; accepted $7^{\text {th }}$ Jun 2017)
}

\begin{abstract}
The purpose of this paper is to propose a new research method to solve the problem of reducing the "source" and "sink" measures in the implementation of low carbon governance, promote the development trend of "reducing sources and increasing exchange rate", and to achieve an ideal state of negative carbon emissions. This paper is based on the research of low carbon sustainable and carbon - water coupling in ecological environment management, and based on the social and economic demands, a carbon cycle system based on carbon emission and carbon capture characteristics and a water resource circulation system characterized by ecological water demand and water pollution are constructed, and the two structures are coupled to each other. The regional carbon and water symbiotic coupling system with the coupling relationship of carbon water circulation system is proposed. This paper analyzes the internal components of carbon-water coupling system and its influencing factors from the perspective of "low carbon", reveals its internal mechanism and establishes a multi-objective game decision model for water resources regulation in regional carbon-water symbiosis system. At the same time, this paper has optimized the water resources regulation and control in the pilot area. In this paper, by adjusting and perfecting the configuration of carbon and water coupling system in the regional water resources system, the "source sink" integration in the carbon cycle is realized, which is the ideal mode of "reducing source and increasing exchange rate".
\end{abstract}

Keywords: comprehensive evaluation model; sustainable development index; carbon-water coupling; low carbon mode; water resource rational allocation

\section{Introduction}

Climate change has a profound impact on the supply and demand of water resources, leading to lower carbon capture capacity of ecosystems; In addition, socio-economic development leads to an increase in carbon emissions, carbon emissions increased and reduced carbon capture resulting in an integrated carbon-water system imbalance (Hannah et al., 2004; Hashem et al., 2016; Mi et al., 2014; Jiang et al., 2016; Petts et al., 2006). Although carbon emission reduction technology has been gradually promoted and applied (IPCC, 2007; Ge et al., 2015; Liu and Liu, 2010; Schramm, 2015), the ecological planning based on returning farmland to forest has also been implemented. But at present, the net carbon emissions are still in a high stage of increase, only from the past "increase sources, reduce sinks" model to "source reduction" model change, has not yet completely transition to the "source reduction and increase sinks" model. The reason is that the mitigation measures of climate change mitigation at the present stage will be separated from "reducing sources" and "increasing sinks" (China Water Resources Bulletin (1998-2007)), and lacking the comprehensive regulation and management system and methods of ecological and environmental systems of "reducing sources" and "increasing sinks". Water is a crucial environmental factor in the carbon capture and storage system of the ecological environment system. The "natural-artificial" dual water cycle changes 


$$
-458-
$$

the regional water conditions, affecting the formation, development and succession of ecosystems, and directly affects the carbon emissions and carbon capture processes of ecosystems, affecting the net carbon emissions of regional carbon (Feng et al., 2016; Zhang, 2016). According to Figure 1 that the economic and social system is carbon source, the ecosystem is carbon sink. According to statistics, compared with 2000, China's carbon emissions and water consumption in 2012 respectively increased by $112.8 \%$ and $7.4 \%$ (Peng et al., 2015; National Development and Reform Commission, (2009)). Increased demand for water resources and increased demand for "fossil fuels" by socio-economic development has resulted in an increase in carbon "source" emissions and a reduction in carbon sinks. Therefore, the conceptual model of carbon-water symbiosis coupling based on carbon-water balance system is constructed, and the factors, structure and mechanism of regional carbon-water coupling system generalization model are discussed. Based on the identification of water resource, water user, carbon source and carbon sink, this paper reveals the relationship between water and water users, the relationship between carbon emission and carbon capture, and the mutual relationship between water cycle and carbon cycle, and to establish a theoretical and applied strategy for carbon-water balance to regulate and control water resources system under the effect of "low carbon".

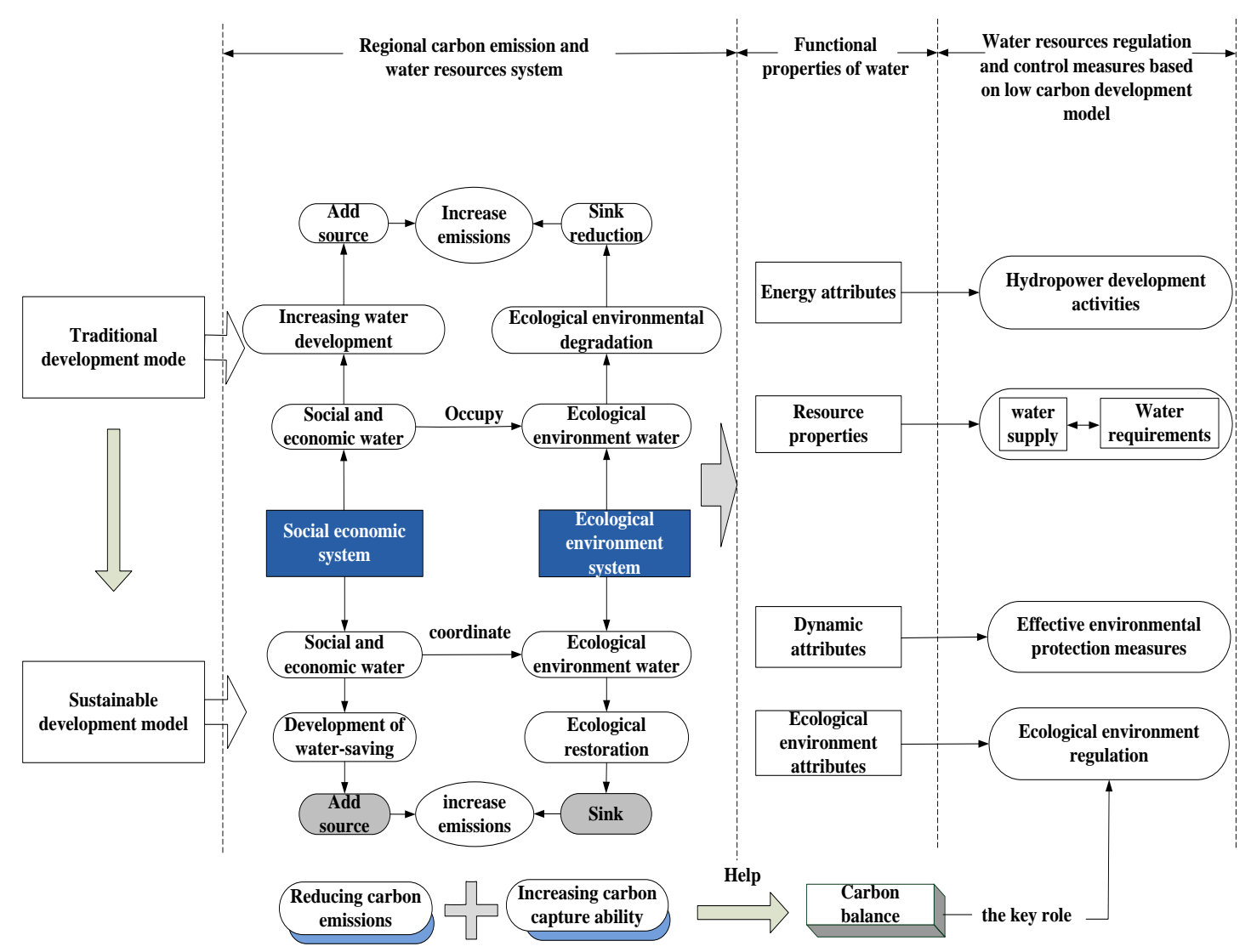

Figure 1. Water resources regulation and control of carbon balance mechanism diagram.

\section{Material and Methods}

In this paper, the water quality and water quantity early warning model of regional carbon and water symbiosis mechanism is established by making use of system modeling, 
data mining, fuzzy evaluation, scenario simulation and game theory. At the same time this paper uses the system optimization decision-making modeling method, combining with multi-objective, uncertain variables of the complex programming mode, to reveal the theory and method of water resources regulation and control based on low carbon model. Specific research methods are as follows:

\section{Analysis and conceptual model of regional water quality and water quantity correlation under low carbon}

From the viewpoint of water resources system, the water circulation system under the regional carbon water symbiosis complex system should include the water quality safety subsystem and the water quantity security subsystem. Water quality security is reflected in if the urban water environment system water quality standards are compliance or not, water security is reflected in whether the water requirement sectors such as ecological environment, living and production in the case of water quality standards are able to achieve the balance between supply and demand. In addition, the regional water resources system is a complex system which takes human construction and operation management as the core. It includes several sub-systems and many components. How to describe the relationship between water quality and quantity and the interaction and evolution of the subsystems is the fundamental question. It is also a key problem to study the interaction mechanism of the water quality and water quantity system in the regional carbon water symbiosis complex system, and to construct the conceptual model of the water quality and quantity correlation system. This part of the study is divided into the following two aspects:

\section{Correlation analysis of regional water quality and water quantity under low carbon model}

Aiming at the structural characteristics of water circulation system with multi-water source, multi-user and multiple transmission and distribution water projects, the water circulation system is decomposed into multiple independent control units by the distributed control method of the complex system. And this paper analyzes the water transport and distribution relations and the pollutant migration and transformation rules of each control unit. The regional carbon water symbiosis complex system was introduced into the analysis of water quality and quantity connection effect. The simulation results are used as the main information source to analyze the regional water quality and water quantity movement mechanism, establish the regional carbon water symbiosis complex system water quality quantity water correlation relations. In practice, researchers will further improve and perfect the joint regulation of water quality and quantity of water resources.

\section{Establishment of the conceptual model of regional water quality and water quantity correlation under low carbon}

It will improve the current water quality studies coupled commonly used "loosely coupled" structure, that is quantity and quality in the system as independent of each subsystem, and then turn the water quality modeling and simulation method. In this paper, we consider the coupling between water quality and quantity, the coupling and interaction between the regional water cycle system and the economic and social water use system from the aspects of the interaction between the artificial - natural binary 
circulation, the integrity of the watershed, the target control at the macroscopic level of the watershed and the concrete simulation of the microcosmic aspect of the river basin and then on the basis of clarifying the rules of water transfer and water quality variation among different parts of the system, a conceptual model of water quality and quantity association system in regional carbon and water symbiosis complex system was established, and the water quality and water quantity correlation system in the study area was comprehensively evaluated, this is the basis of the follow-up research.

\section{Identification of carbon - water symbiosis mechanism and theory and method of system comprehensive evaluation under low - carbon}

At present, the theory and method of rational allocation of water resources based on low carbon development model are still in the exploratory stage, and there is no mature model available at home and abroad for reference. This paper summarizes the domestic and international water resources allocation theory and technology research new progress on the basis of the proposed low carbon development model for the rational allocation of water resources mechanism of carbon water symbiosis system to multi-objective decision-making and simulation technology as the key support, water resource regulation and control model of carbon - water symbiosis system from low carbon perspective. The specific research methods adopted are as follows:

Identification technology of operation mechanism of regional carbon-water symbiosis system under low carbon mode

The prerequisite and basis of regional carbon-water symbiosis system is identification of system configuration, in which the identifying objects include the water circulating system under universal concept and the carbon cycling system based on carbon source and carbon sink (Socio-economic system mainly for the carbon emissions of the system, while the ecosystem is mainly manifested as carbon-capture systems). The core of this part of the research is to draw the model of network generalization of Regional Water Resources System based on the carbon-water symbiosis of coupled system based on the distribution of the existing carbon-water circulating system in the experimental area, based on the system identification of water source, water user, carbon source and carbon sink. The model of network generalization of Regional Water Resources System (shown in Figure 2), through the system network model method to establish the configuration of unit and the node, the system to establish the relationship between water and water users, the relationship between carbon-emissions and carbon-capture and the constraint relationship between water cycle and carbon cycle.

The theory and method of comprehensive evaluation of regional carbon and water symbiosis system in low carbon mode

It is necessary to establish a reasonable and effective evaluation system based on the above-mentioned regional carbon-water symbiosis system, the main task of the research is to study the control planning method and management strategies of water resources under the low carbon perspective with the system optimization and decision analysis method, the main research tasks include: Selecting the evaluation index of the research object, establishing the evaluation mathematical model, determining the key parameters of the model, solving the model and analyzing the result. The selection of evaluation index is the emphases and key point. 


$$
-461-
$$
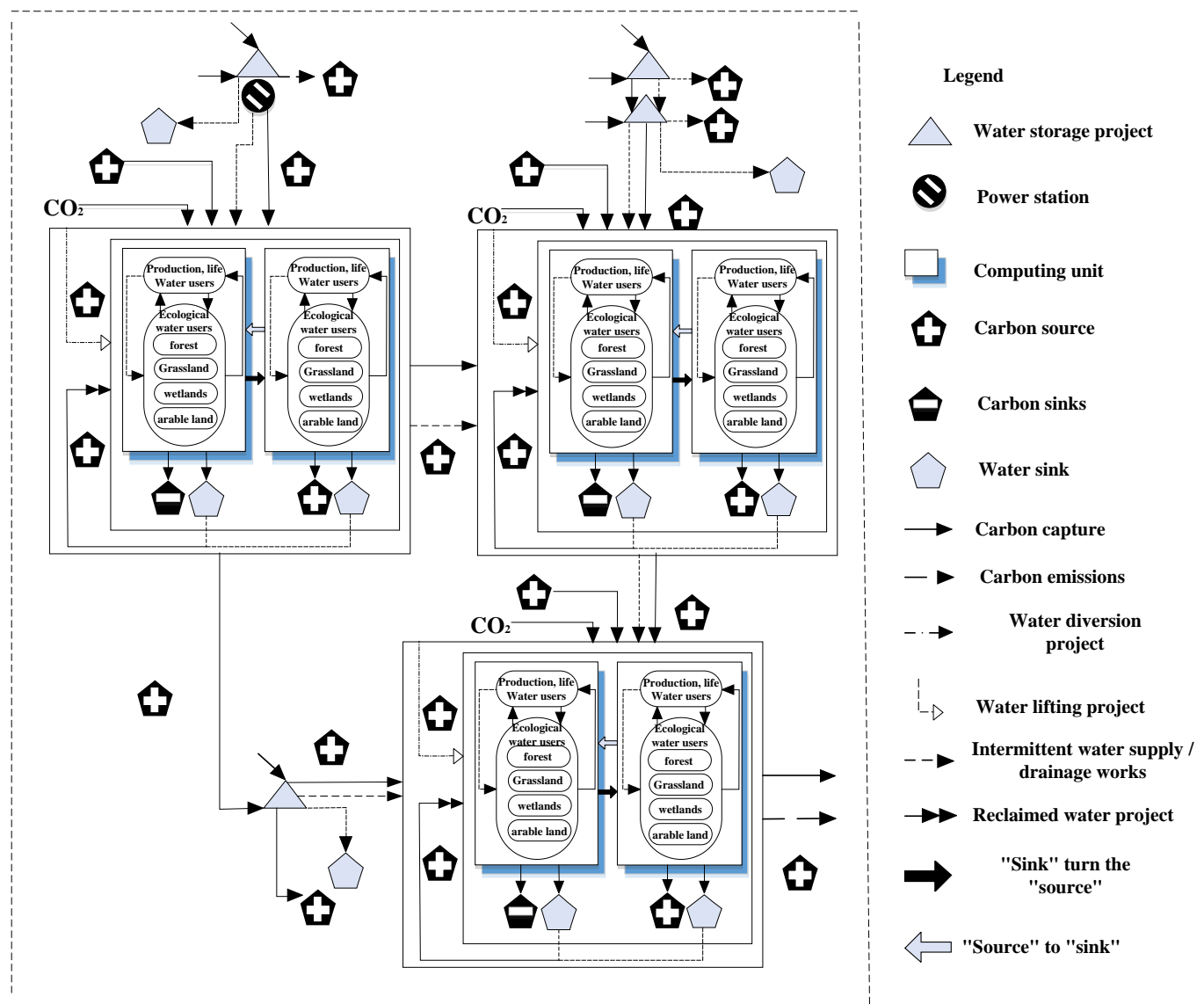

Figure 2. Regional carbohydrate generalized model symbiotic system schematic.

Water quality and water quantity simulation of regional carbon - water symbiosis mechanism and safety early warning technology of water circulation system

Through the analysis of the literature, it is found that the previous studies on the simulation of water pollution are mostly carried out under a single static characteristic, which is not consistent with the objective reality. Because the practical meaning of the water cycle system with "multiple fuzzy" complex features: Uncertainty (Spatial and temporal distribution unevenness of water), Dynamic (The dynamic of regional ground confluence changed follow the time), Irregularity (Irregular nature of socio - economic water use). Therefore, it is reasonable and practicable to study the regional water quality simulation and early warning system in combination with the occurrence, development and spread of dynamic evolution disaster under the "multiple fuzzy" characteristics mentioned above.

Construction of water quality and quantity simulation model based on scenario simulation of regional carbon and water symbiosis mechanism

First of all, it is to collect extensively with all types of hydrological data which based on the test area, and regional geographical environment, the main water supply facilities, drainage network and channels, precipitation and other spatial and temporal distribution of data. And at the same time to identify the influencing factors and processes of water quality and water safety under the regional carbon and water symbiosis mechanism; 
Based on the historical and literature data, the multiple fuzzy features of the main driving factors of the system are analyzed; On this basis, the uncertainty assumption method is used to set up hypothetical condition of the evolution of the environment, which can be used as the input of water modeling and water pollution simulation model construction of regional carbon-water symbiosis mechanism, and systematically identify water sources, pollution sources, carbon "sources" and carbon sinks, and establish allocation units and nodes to establish a database of water sources and pollution sources, carbon emissions and carbon capture, and water cycle and carbon cycle system interaction and status information, and combined with the Petri Net rule model described as the scene data source generation tool, as the establishment of water quality and water quality of the set of elements of the background information.

Pre-warning technology of water quality and water security system based on scenario prediction model of regional carbon-water symbiosis mechanism

Finding the historical water quality and quantity database of the experimental area and using the multi-dimensional correlation function to solve the problem of nonlinear mapping between the water security risk factors and the key early warning indicators, and obtain the connection function of the water quality and water quality between risk factors and warning indicators through the fitting calculation; Based on the historical data, the safety factor and the early warning classification correlation model were established to determine the water pollution classification type, the early warning control parameter and the key early warning index threshold ; The establishment of a diagnostic model will change with the diffusion time of the key indicators of police intelligence and early warning indicators to assess the threshold value of tracking and comparison, once the alarm threshold is exceeded signal (Figure 3), and input to the corresponding early warning level deduction model to realize the logical structure of early warning response, and provide the guarantee for regional water resources regulation and control.

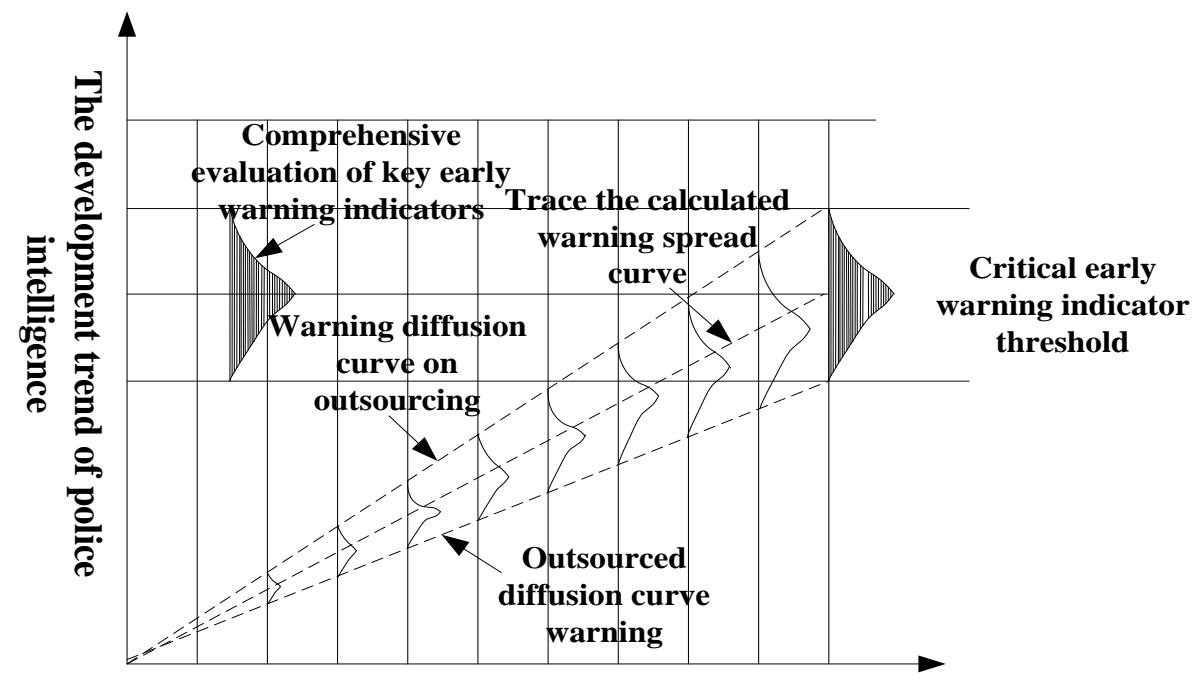

Diffusion time

Figure 3. Key early warning indicators threshold curve based on tracking comparison. 


\section{Regulation and Control of Water Quality and Quantity in Regional Carbon and Water Symbiosis System from Low Carbon Perspective}

Regional carbon - water symbiosis mechanism identification and comprehensive evaluation of the system and safety pre-warning of the water cycle system provide a basis for the regulation and control of water resources based on the coupling mechanism of regional water quality and water quantity under low carbon mode. However, how to plan and manage regional carbon-water symbiosis system to optimize the water supply regulation and water pollution control of regional water management departments to determine whether the carbon-water circulation coupling system of the study area is balanced, and to determine compliance with regional water bearing capacity safety standards is a regional carbon water symbiosis system water quality and water regulation and control of another important prerequisite. This article mainly studies according to the following two aspects.

A multi-objective game decision-making model of water quality and water quantity regulation in regional carbon water symbiosis system under low carbon perspective

Considering the economic, environmental and social factors of the test area, this paper puts forward the idea and method of constructing a multi-objective game decision-making model of water quality and water quantity regulation in regional carbon water symbiosis system under low carbon perspective, and designs the model structure based on multi-objective game analysis and the model solving method of co-evolutionary algorithms, and selects the water resources system of the typical city in the test area to model. Based on the theory knowledge and technology of system, optimization decision, modeling method comprehensively, this paper adopts the method of multi-objective decision-making and simulation and uses decision-making and simulation methods to set middle and long-term water resources regulation sequence elements, and gives water resources allocation under different scenarios of supply and demand, water quality control and carbon emission reduction.

Formulating regulation and control measures of water quality and water quantity regulation in regional carbon water symbiosis system under low carbon perspective

Based on regulation and control methods of water resources in regional carbon water symbiosis system under low carbon perspective is a multi-source, multi-carbon source and multi-control factors integrated water resources management model. In this paper, the water resources regulation model based on low carbon development model is used to analyze the schemes, optimize the comparison and output the regulation scheme. Then, according to the key assumptions of the carbon-water symbiosis system, the results are used to construct the system evaluation and evaluation system, and the recommended scheme is analyzed and evaluated. Then the article gets the recommended governance solution. Finally, based on regional general situation, this paper puts forward practical and feasible measures to ensure water quality and water quality of regional carbon-water symbiosis system under low carbon perspective. 


\section{Results and Discussion}

First of all, this paper analyzes the correlation and transformation between the various components stem from the components of the regional water quality and water quantity system and its influencing factors. In order to establish the conceptual model of regional water quality and water quantity system and reveals its internal influencing mechanism, this provides the base of qualitative and quantitative research for carbon water symbiosis system. Secondly, on the basis of the characteristics of carbon-water coupling system, under the principle of "low carbon" and "sustainable", considering various factors that "Human - Carbon - Water" Realistic Environment has an impact on the safety and sustainability of regional water resources systems. The effect of multi - factors on carbon - water symbiosis system was discussed, and the mechanism of regional ecological, environmental, economic and social systems under low carbon mode was revealed. Furthermore, the constituent elements of the regional water resources system and their influencing factors were proposed according to the carbon-water symbiosis system model. The interaction between the components was analyzed and the water quality prediction model based on water pollution simulation and evaluation was established. , To improve the water supply security, water security. Finally, through the modeling and case analysis of the regulation of water resources in regional carbon - water symbiosis system, this paper reveals the mechanism of Regional Carbon-Water Coupling System and the improvement of water resources regulation and control measures under the View of "low carbon", and providing a scientific and reliable technical support for the river basin water system governance.

\section{Conclusion}

In this paper, the Haihe basin is considered as a research area, taking the conceptual model and the role identification of carbon-water coupling system into account. Through systematic evaluation and simulation, the characteristics of carbon and water cycle and water resources system are identified, and the regional carbon emission benefit, carbon capture efficiency and carbon emission net benefit divided by basin were focused. Design and establishment of water security early warning model under the low carbon effect model, in order to meet the requirements of water resources management and climate change comprehensive response under the conditions of low carbon and water security, this paper, based on the water security coordination control method in the field of environmental governance, scientifically allocates the water resources of the river basin. Finally, this paper presents a new idea and new method to solve the water resources regulation and control in the carbon-water symbiosis system.

Acknowledgements. This research is supported by the Project of Tianjin Science and Technology Plan (Grant No. 15ZLZLZF00410) and Humanities and Social Science Foundation of Ministry of Education of China (Grant No. 12YJC630248) and the Opening Foundation of Tianjin University State Key Laboratory of Hydraulic Engineering Simulation and Safety (Grant No. HESS-1701) and Social Science Foundation of the Ministry of Education of China (Grant No. 14YJC630211) and the Social Science Foundation of China (Grant No. 15BGL173). 


\section{REFERENCES}

[1] China Water Resources Bulletin (1998-2007). China Water Conservancy and Hydropower Press.

[2] Feng, Z.X., et al. (2016): The recognition of multi-class protein folds by adding average chemical shifts of secondary structure elements. - Saudi Journal of Biological Sciences 23(2): 189-197.

[3] Ge, S., et al. (2015): TD-GC-MS Analysis on Antibacterial and Safety Characteristics of Volatiles from Bamboo during Cavitation, Carbonization and Activation for Public Health. - Journal of Pure and Applied Microbiology 9(3): 1907-1912.

[4] Hannah, D.M., Wood, P.J., Sadler, J.P. (2004): Ecohydrology and hydroecology: A 'new paradigm'. - Hydrological Process 18: 3439-3445.

[5] Hashem, A., et al. (2016): Alleviation of cadmium stress in Solanum lycopersicum L. by arbuscular mycorrhizal fungi via induction of acquired systemic tolerance. - Saudi Journal of Biological Sciences 23(2): 272-281.

[6] IPCC (2007). Summary for Policymakers. Climate Change 2007: Mitigation. Report of Working Group III of the Fourth Assessment Report of the Intergovernmental Panel on Climate Change. - Cambridge University Press, Cambridge, United Kingdom.

[7] Jiang, G.J., Lv, H.D., Liu, L. (2016): Process safety evaluation model based on LEC and Grey Theory. - Journal of Mechanical Engineering Research and Developments 39(1): 24-29.

[8] Liu, H.B., Liu, Z.L. (2010): Recycling Utilization Patterns of Coal Mining Waste in China. - Resources, Reservation and Recycling (12): 1331-1340.

[9] Mi, C., et al. (2014): A novel experimental teaching approach for electrical engineering based on semi-physical simulation. - World Transactions on Engineering and Technology Education 12(4): 779-783.

[10] National Development and Reform Commission (2009). Energy Bureau, Evaluation of Socioeconomic Impacts of Mitigating Climate Change, 30-70.

[11] Peng, W., et al. (2015): Separation of hemicelluloses from eucalyptus species: investigating the residue after alkaline treatment. - Cellulose Chemistry and Technology 49(9-10): 757-764.

[12] Petts, G., Morales, Y., Sadler, J. (2006): Linking hydrology and biology to assess the water needs of river ecosystems. - Hydrological Process 20: 2247-2251.

[13] Schramm D.H. (2015): Current and future trends in the automotive industry. - Renewable Energy and Sustainable Development 1(2): 233-234.

[14] Zhang, X.Y. (2016): Optimized design of adhesive formations for tennis ball. - Journal of Mechanical Engineering Research and Developments 39(1): 217-225. 